\title{
Clinical Outcomes of the Intraluminal Approach for Long Occlusive Femoropopliteal Lesions Assessed by Intravascular Ultrasound
}

\author{
Shinsuke Mori, Keisuke Hirano, Yoshiaki Ito, Masahiro Yamawaki, Motoharu Araki, Norihiro Kobayashi, \\ Hideyuki Takimura, Yasunari Sakamoto, Masakazu Tsutsumi, Takuro Takama, Yohsuke Honda, \\ Takahiro Tokuda, Kenji Makino and Shigemitsu Shirai
}

Department of Cardiology, Saiseikai Yokohama City Eastern Hospital, Yokohama, Japan

Aim: To investigate the relationship between intravascular ultrasound (IVUS) findings and restenosis after stent implantation for long occlusive femoropopliteal (FP) lesions using the intraluminal approach.

Methods: This was a single-center retrospective study of 45 patients (49 lesions) with de novo long occlusive FP lesions treated with bare metal stents implanted using the intraluminal approach under IVUS guidance from April 2007 to December 2014. All patients were followed up at least 12 months. The preprocedural and postprocedural IVUS findings were compared for patients with and without restenosis, which was defined as a peak systolic velocity ratio of $>2.4$ on duplex ultrasonography or $>50 \%$ diameter stenosis on angiography.

Results: Within 12 months, 13 patients (14 lesions) developed restenosis, whereas 32 patients (35 lesions) did not (restenosis rate $=29 \%$ ). The male:female ratio and the prevalence of diabetes mellitus, hemodialysis, and critical limb ischemia were similar between the two groups. No significant differences were observed in lesion length, chronic total occlusion (CTO) length, and the percentage of involving popliteal lesion between the two groups. A whole intraplaque route was gained in 15 lesions $(31 \%)$. Multivariate analysis revealed that the within-CTO intramedial route proportion and the distal lumen cross-sectional area (CSA) were independent predictors of restenosis. Receiver operating characteristic analysis showed that the best cutoff values of these parameters were $14.4 \%$ and $17.7 \mathrm{~mm}^{2}$, respectively.

Conclusions: In patients with long occlusive FP lesions undergoing stent placement using the intraluminal approach, a whole intraplaque route was gained in $31 \%$. Restenosis is more likely if IVUS shows a within-CTO intramedial route proportion of $>14.4 \%$ or distal lumen CSA of $<17.7 \mathrm{~mm}^{2}$.

See editorial vol. 24: 473-474

Key words: Intraluminal approach, Femoropopliteal artery, Intravascular ultrasound, Chronic total occlusion

Copyright@2017 Japan Atherosclerosis Society

This article is distributed under the terms of the latest version of CC BY-NC-SA defined by the Creative Commons Attribution License.

\section{Introduction}

Endovascular treatment (EVT) is less invasive and safer, and it is regarded as a first-line therapy for

Address for correspondence: Shinsuke Mori, Department of Cardiology, Saiseikai Yokohama City Eastern Hospital, 3-6-1

Shimosueyoshi, Tsurumi-ku, Yokohama City Kanagawa 230-

0012 Japan

E-mail: hello_morisun@yahoo.co.jp

Received: June 6, 2016

Accepted for publication: August 10, 2016 femoropopliteal (FP) lesions ${ }^{1,2}$. For EVT of complex lesions, such as TransAtlantic Inter-Society Consensus (TASC II) types $\mathrm{C}$ or $\mathrm{D}$, the primary patency rate at 12 months after self-expandable stent placement is reportedly $66 \%-78 \%{ }^{3-6)}$, which is acceptable. The intraluminal and subintimal approaches are selected in EVT for long occlusive lesions. Although the clinical outcomes after the subintimal approach for long occlusive lesions have been reported ${ }^{6-9)}$, relatively few studies have investigated the clinical outcomes after the intraluminal approach for such lesions, and the 
outcomes of the intraluminal approach has not yet been accurately evaluated by intravascular ultrasound (IVUS). Therefore, the aim of this study was to evaluate the relationship between preprocedural and postprocedural IVUS findings and restenosis at 12 months after stent implantation for long occlusive lesions using the intraluminal approach.

\section{Methods}

\section{Patients}

This was a non-randomized, retrospective, singlecenter study. Between April 2007 and December 2014, EVT was performed for 925 de novo FP lesions at our hospital, of which 876 were excluded because of acute/subacute lower-limb ischemia $(n=12)$, stenotic or short $(<10 \mathrm{~cm})$ occlusive lesions $(n=660)$, EVT by the subintimal approach $(n=12)$, procedural failure $(n=14)$, EVT with angioplasty alone $(n=46)$, non full covered stents $(n=14)$, disuse of IVUS $(n=80)$, use of drug eluting stent (DES) $(n=8)$, death/major amputation/dropout within 12 months $(n=26)$, or inadequate IVUS data $(n=4)$. Therefore, 45 patients (49 lesions) who underwent bare metal stent (BMS) implantation for long occlusive FP lesions (occlusion length $>10 \mathrm{~cm}$ ) using the intraluminal approach were included for analysis (Fig. 1). Restenosis according to duplex ultrasonography or angiographic criteria was observed in 14 lesions within 12 months (restenosis rate $=29 \%$ ). These 45 patients were divided into two groups: restenosis and non-restenosis groups. All patients were on exercise and drug therapy, and had symptoms corresponding to categories $2-6$ of the Rutherford classification ${ }^{1)}$. When angiography revealed occlusion of the FP vessels, vascular specialists (including vascular surgeons and interventional cardiologists) decided whether EVT was appropriate. The present study was conducted in accordance with the tenets of the Declaration of Helsinki and all patients submitted informed consent to participate in this study.

\section{Interventions}

The crossover or ipsilateral approach was used for EVT. 6-Fr or 7-Fr sheaths were inserted and unfractionated heparin $(5000 \mathrm{U})$ was injected intraarterially. A microcatheter or an over-the-wire balloon was used as a support for advancing a 0.014-inch guidewire to the occluded site. The procedure was performed such that the guidewire was passed through the true lumen of the vessel as much as possible, as confirmed by body surface echography and IVUS, as needed. In cases in which it was not possible to pass the guidewire antegradely, we switched to a retrograde-approach via the popliteal or tibial artery ${ }^{9,10)}$.
After a 0.014-inch guidewire was passed through the target lesion, IVUS images were recorded by manual pullback through the study segment at a uniform and steady rate. If it was not possible to cross the lesion with the IVUS catheter, balloon dilatation was performed with a 3-mm-diameter balloon prior to IVUS recording. A second attempt to access the intraplaque route was up to the discretion of the operator when the guidewire was passed through the subintimal or intramedial route. BMSs (Zilver 518 stent; Cook Medical, Bloomington, IN, USA; S.M.A.R.T stent; Cordis Endovascular, Warren, NJ, USA) were implanted so that it fully covered the lesion, meaning that the stents were implanted from angiographic normal-to-normal segments. A stent with a diameter 1-2 mm larger than the reference vessel diameter proximal to the target lesion was selected. When two or more stents were used for a long lesion, the overlap was $\leq 10 \mathrm{~mm}$. Predilatation and postdilatation was performed routinely. At the end of the procedure, IVUS images were recorded again using commercially available IVUS consoles (s $5^{\mathrm{TM}}$ Imaging System; Volcano Corporation, Rancho Cordova, CA, USA or VISIWAVE ${ }^{\mathrm{TM}}$; Terumo Corporation, Tokyo, Japan) and a phased-array 20-MHz IVUS catheter (Eagle Eye Gold; Volcano Corporation) or $35-\mathrm{MHz}$ IVUS catheter (View it; Terumo Corporation).

\section{Medical Therapy}

Dual antiplatelet therapy (DAPT) with aspirin (100 mg/day) plus clopidogrel $(75 \mathrm{mg} /$ day), ticlopidine $(200 \mathrm{mg} /$ day $)$, or cilostazol $(200 \mathrm{mg} /$ day $)$ was started at least 3 days before stenting and was continued for at least 2 months afterward.

\section{IVUS Analysis}

For analysis of the IVUS data, the $s 5^{\mathrm{TM}}$ Imaging System (Volcano Corporation) and VISIWAVE ${ }^{\text {TM }}$ (Terumo Corporation) were used. Two experienced observers who were unaware of the clinical and angiographic findings performed all IVUS analyses. The IVUS parameters measured or calculated were route of chronic total occlusion (CTO) (intraplaque, subintimal, and intramedial) ${ }^{11)}$, maximum calcification arc, maximum and minimum stent cross-sectional area (CSA), proximal and distal CSA of the external elastic membrane (EEM), luminal CSA, plaque burden, reference luminal CSA, reference diameter, stent/reference diameter ratio, stent expansion ratio, radial stent symmetry index, and axial stent symmetry index. The proximal and distal reference segments selected for analysis were the most normal-looking cross-sections within $10 \mathrm{~mm}$ of the proximal and distal margins of the stent before the origin of any large side branches ${ }^{12)}$. 
925 lesions

Underwent EVT for de novo FP lesions

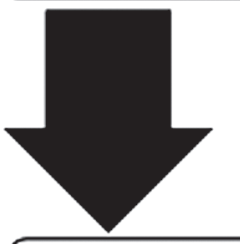

684 excluded

12 acute/subacute lower limb ischemia

660 stenosis or occlusion length less than $10 \mathrm{~cm}$

12 subintimal approach

\section{1 lesions}

Underwent EVT for long occlusive FP lesions by intraluminal approach

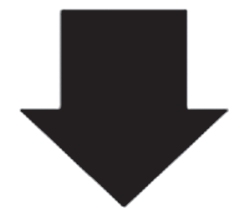

74 excluded

14 procedural failure (success rate $94.2 \%$ )

46 balloon angioplasty alone

14 non full covered stent

\section{7 lesions}

Underwent full covered stent placement for long occlusive FP lesions by intraluminal approach

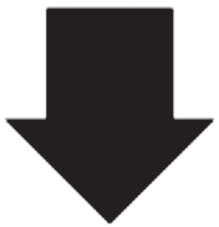

118 excluded

80 disuse of IVUS, 8 DES use

16 death within 12 months

4 major amputation within 12 months

6 drop out within 12 months, 4 inadequate IVUS data

\section{9 lesions (45 patients)}

Underwent full covered BMS placement for long occlusive FP lesions with adequate IVUS data by intraluminal approach

Fig. 1. Study Flowchart

EVT: endovascular therapy.

FP: femoropopliteal.

IVUS: intravascular ultrasound.

DES: drug eluting stent.

BMS: bare metal stent.

Stent-edge dissection was also investigated. The proportion of stents placed using the intraplaque route within the CTO was calculated as IVUS flame numbers of the guidewire and IVUS transducer being located via the intraplaque route divided using the total IVUS flame number of the whole CTO route. The proportions of placements via the subintimal/ intramedial routes were calculated in the same manner.

\section{Definitions}

Diabetes mellitus (DM) was defined as a condition requiring treatment with oral hypoglycemic drugs or insulin, or as a casual plasma glucose concentration of $>200 \mathrm{mg} / \mathrm{dl}$ or HbA1c of $>6.5 \%$. Chronic kidney disease was defined as a glomerular filtration rate of $<60 \mathrm{ml} / \mathrm{min} / 1.73 \mathrm{~m}^{2}$. Coronary artery disease was defined as documented angina pectoris, previous myocardial infarction, or a history of coronary revascularization. Cerebrovascular disease was defined as previ- 
Table 1. Patient Characteristics

\begin{tabular}{|c|c|c|c|c|}
\hline Variables & $\begin{array}{c}\text { Total } \\
(n=45)\end{array}$ & $\begin{array}{l}\text { Restenosis } \\
\quad(n=13)\end{array}$ & $\begin{array}{l}\text { Non-restenosis } \\
\quad(n=32)\end{array}$ & $p$ value \\
\hline Age > 70 years $(\%)$ & $23(51)$ & $4(31)$ & $19(59)$ & 0.08 \\
\hline Hypertension (\%) & $41(91)$ & $13(100)$ & $28(88)$ & 0.18 \\
\hline Dyslipidemia (\%) & $20(44)$ & $5(38)$ & $15(47)$ & 0.61 \\
\hline Chronic renal disease (\%) & $26(58)$ & $10(77)$ & $16(50)$ & 0.09 \\
\hline Dialysis $(\%)$ & $8(18)$ & $4(31)$ & $4(13)$ & 0.15 \\
\hline Coronary artery disease (\%) & $14(31)$ & $4(31)$ & $10(31)$ & 0.97 \\
\hline Cerebrovascular disease (\%) & $5(11)$ & 0 & $5(16)$ & 0.13 \\
\hline $\mathrm{EF}<40 \%(\%)$ & $2(4)$ & $1(8)$ & $1(3)$ & 0.50 \\
\hline Thienopyridines (\%) & $35(78)$ & $10(77)$ & $25(78)$ & 0.93 \\
\hline Cilostazol (\%) & $16(34)$ & $5(38)$ & $11(34)$ & 0.80 \\
\hline No. of anti-platelets & $2.1 \pm 0.5$ & $2.1 \pm 0.6$ & $2.1 \pm 0.5$ & 0.94 \\
\hline Warfarin $(\%)$ & $10(22)$ & $5(38)$ & $5(16)$ & 0.09 \\
\hline Statin $(\%)$ & $17(38)$ & $3(23)$ & $14(44)$ & 0.19 \\
\hline Continuation of DAPT at 2 months (\%) & $40(89)$ & $11(85)$ & $29(91)$ & 0.56 \\
\hline
\end{tabular}

EF: ejection fraction

DAPT: dual antiplatelet therapy

ous stroke, transient ischemic attacks, carotid artery revascularization, or intracranial hemorrhage ${ }^{13)}$. The definition of critical limb ischemia was the same as described elsewhere ${ }^{14)}$. Below-the-knee (BTK) runoff was assessed by angiography after stent placement. Poor runoff was defined as runoff in one or no BTK vessel ${ }^{15)}$. The intraluminal approach indicates EVT using a 0.014-inch or 0.018-inch guidewire with a supporting catheter ${ }^{7)}$. Stenting was classified as successful if angiography demonstrated that residual stenosis was $\leq 30 \%$ without flow-limiting dissection ${ }^{16}$. Restenosis was defined as a peak systolic velocity ratio of $>2.4$ on duplex ultrasonography or $>50 \%$ diameter stenosis or occlusion on quantitative vascular angiography ${ }^{17)}$. Target lesion revascularization (TLR) was defined as the need for repeated procedures (endovascular or surgical) because of a problem arising from the lesion $(+1 \mathrm{~cm}$ proximally and distally to include edge phenomena $)^{6}$. . The definition of stent thrombosis was the same as described elsewhere ${ }^{18)}$. The definitions of IVUS parameters followed those of a previous report ${ }^{19)}$. Wire passage in occlusive lesions was classified according to IVUS findings: intraplaque, inside the plaque; subintimal, between the plaque and media; and intramedial, between the media and adventitia ${ }^{11)}$. Stent-edge dissection was defined as dissection at a stent edge $(<5 \mathrm{~mm}$ proximal or distal to the stent margin) ${ }^{16,20)}$.

\section{Clinical Follow-Up}

Postprocedural evaluation of symptoms, measurement of the ankle-brachial index (ABI), and duplex ultrasonography were performed at $1,3,6,9$, and 12 months after stent placement. When duplex ultrasonography suggested restenosis, angiography was performed for confirmation.

\section{Statistical Analysis}

Statistical analysis was performed using JMP software (version 10.0.0, SAS Institute, Cary, NC, USA). Continuous variables with normal distributions are presented as the means \pm standard deviations. Categorical data are presented as frequencies. Continuous variables were compared using the unpaired Student's $t$-test or the Mann-Whitney $U$-test. Categorical data were compared with the chi-squared test or Fisher's exact test. Univariate and multivariate logistic regression analysis were performed to identify predictors of restenosis. Variables with a probability $(p)$ value of $<0.05$ on univariate analysis were entered into a multivariate regression model. Receiver operating characteristic (ROC) analysis was performed to determine 
Table 2. Lesion and Procedural Characteristics

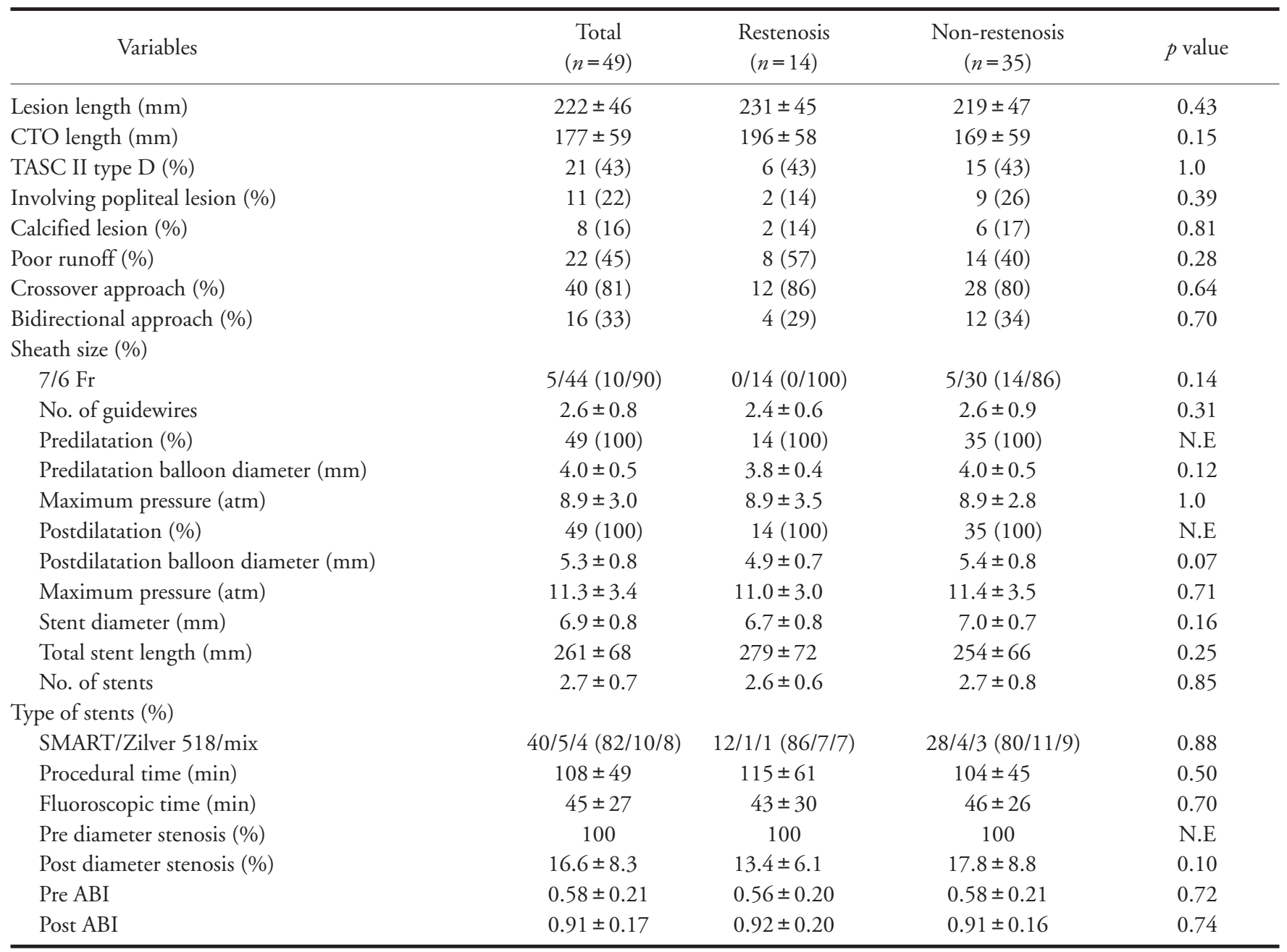

CTO: chronic total occlusion

TASC: trans-Atlantic Intersociety Consensus.

ABI: ankle-brachial index.

the optimal cutoff value of the within-CTO intramedial route proportion and distal lumen CSA to predict restenosis at 12 months after stent placement, with each cutoff point being selected to yield the highest value for the sum of sensitivity and specificity. The area under the ROC curve (AUC) was used as a measure of the accuracy of each parameter. In all analyses, $p$ values of $<0.05$ were considered statistically significant.

\section{Results}

\section{Patients and Procedural Characteristics}

In the present study, 49 lesions in 45 patients were investigated. Restenosis was detected in 14 lesions (29\%) at 12 months after stent placement. Baseline characteristics are shown in Table 1. There were no significant differences in the percentage of patients aged $>70$ years; male:female ratio; or prevalence of $\mathrm{DM}$, dialysis, and critical limb ischemia between the two groups. Regarding medications, cilostazol use and continuation of DAPT at 2 months were also similar between the two groups. Baseline lesion and procedural characteristics are shown in Table 2. A crossover approach was chosen in 40 lesions $(81 \%)$ and a 6 Fr sheath was most commonly used (90\%). A bidirectional approach was used in 16 lesions (33\%). The percentages of TASC II type D lesions and lesions extending to the popliteal artery were $43 \%$ and $22 \%$, respectively. Mean lesion length, CTO length, and total stent length were $222 \pm 46,177 \pm 59$, and $261 \pm$ $68 \mathrm{~mm}$, respectively. There were no significant differences in these variables between the two groups. 
Table 3. IVUS findings

\begin{tabular}{|c|c|c|c|c|}
\hline Variables & $\begin{array}{c}\text { Total } \\
(n=49)\end{array}$ & $\begin{array}{c}\text { Restenosis } \\
(n=14)\end{array}$ & $\begin{array}{l}\text { Non-restenosis } \\
\quad(n=35)\end{array}$ & $p$ value \\
\hline Maximum arc of calcification (degree) & $126 \pm 147$ & $129 \pm 145$ & $124 \pm 150$ & 0.93 \\
\hline Intraplaque (\%) & $73 \pm 28$ & $60 \pm 32$ & $78 \pm 26$ & 0.0498 \\
\hline Subintimal (\%) & $18 \pm 19$ & $24 \pm 21$ & $15 \pm 18$ & 0.17 \\
\hline \multicolumn{5}{|l|}{ Reference segment } \\
\hline Proximal EEM CSA $\left(\mathrm{mm}^{2}\right)$ & $54.2 \pm 22.0$ & $54.5 \pm 23.1$ & $54.1 \pm 21.9$ & 0.95 \\
\hline Proximal lumen CSA $\left(\mathrm{mm}^{2}\right)$ & $29.3 \pm 12.7$ & $28.6 \pm 10.5$ & $29.6 \pm 13.6$ & 0.80 \\
\hline Distal EEM CSA $\left(\mathrm{mm}^{2}\right)$ & $31.6 \pm 9.8$ & $28.3 \pm 9.5$ & $32.9 \pm 9.7$ & 0.13 \\
\hline Distal lumen CSA $\left(\mathrm{mm}^{2}\right)$ & $17.9 \pm 5.7$ & $15.2 \pm 4.7$ & $19.0 \pm 5.8$ & 0.0312 \\
\hline Axial symmetry index & $0.53 \pm 0.13$ & $0.54 \pm 0.14$ & $0.53 \pm 0.12$ & 0.78 \\
\hline Stent expansion ratio & $0.59 \pm 0.19$ & $0.59 \pm 0.18$ & $0.59 \pm 0.19$ & 0.94 \\
\hline Stent/vessel diameter & $1.31 \pm 0.17$ & $1.30 \pm 0.17$ & $1.31 \pm 0.17$ & 0.98 \\
\hline Stent-edge dissection & $3(6)$ & $2(14)$ & $1(3)$ & 0.13 \\
\hline
\end{tabular}

IVUS: intravascular ultrasound.

CTO: chronic total occlusion.

CSA: cross-sectional area.

EEM: external elastic membrane.

\section{IVUS Findings}

Preprocedural and postprocedural IVUS findings are detailed in Table 3. The percentages of the intraplaque, subintimal, and intramedial routes were $73 \% \pm$ $28 \%, 18 \% \pm 19 \%$, and $9 \% \pm 14 \%$, respectively. The whole intraplaque route was gained in 15 lesions (31\%). The proportion of the intraplaque route was significantly lower and the proportion of the intramedial route was significantly higher in the restenosis group $(60 \% \pm 32 \%$ vs. $78 \% \pm 26 \%, p<0.05$, and $16 \% \pm 14 \%$ vs. $7 \% \pm 13 \%, p<0.05$, respectively). The maximum and minimum stent CSA were similar between the two groups $\left(22.8 \pm 5.5\right.$ vs. $24.4 \pm 6.8 \mathrm{~mm}^{2}, p=0.43$, and $12.3 \pm 2.8$ vs. $13.2 \pm 3.5 \mathrm{~mm}^{2}, p=0.36$, respectively). There were no significant differences in the proximal EEM CSA and proximal lumen CSA, and the distal EEM CSA $\left(54.5 \pm 23.1\right.$ vs. $54.1 \pm 21.9 \mathrm{~mm}^{2}$, $p=0.95,28.6 \pm 10.5$ vs. $29.6 \pm 13.6 \mathrm{~mm}^{2}, p=0.80$, and $28.3 \pm 9.5$ vs. $32.9 \pm 9.7 \mathrm{~mm}^{2}, p=0.13$, respectively). However, the distal lumen CSA was significantly smaller in the restenosis group $(15.2 \pm 4.7$ vs. $19.0 \pm$ $\left.5.8 \mathrm{~mm}^{2}, p<0.05\right)$. The radial symmetry index, axial symmetry index, stent expansion ratio, stent/vessel diameter, and the rate of stent-edge dissection were similar between the two groups.

\section{Univariate and Multivariate Logistic Regression Analysis of Restenosis}

Univariate and multivariate logistic regression analysis were performed to identify independent predictors of restenosis. Within-CTO intramedial route proportion and distal lumen CSA were entered into the multivariate model. Within-CTO intraplaque route proportion was not entered into that because of the associations with the within-CTO intramedial route proportion. As shown in Table 4, on multivariate analysis, within-CTO intramedial route proportion [odds ratio $(\mathrm{OR})=1.05 ; 95 \%$ confidence interval $(\mathrm{CI})=1.01-1.11, p<0.05]$ and distal lumen CSA $(\mathrm{OR}=0.85 ; 95 \% \mathrm{CI}=0.72-0.98, p<0.05)$ were independent predictors of restenosis.

\section{ROC Analysis}

The results of ROC analysis of within-CTO intramedial route proportion and distal lumen CSA are shown in Fig. 2. The AUC for within-CTO intramedial route proportion was $0.691(95 \% \mathrm{CI}=0.519-$ $0.863, p<0.05)$ and the best cutoff value of withinCTO intramedial route proportion to predict restenosis within 12 months after stent placement was $14.4 \%$, which had a sensitivity of $64.3 \%$ and a specificity of $80.0 \%$. In the present series, the within-CTO 
Table 4. Logistic Regression Analysis of Restenosis

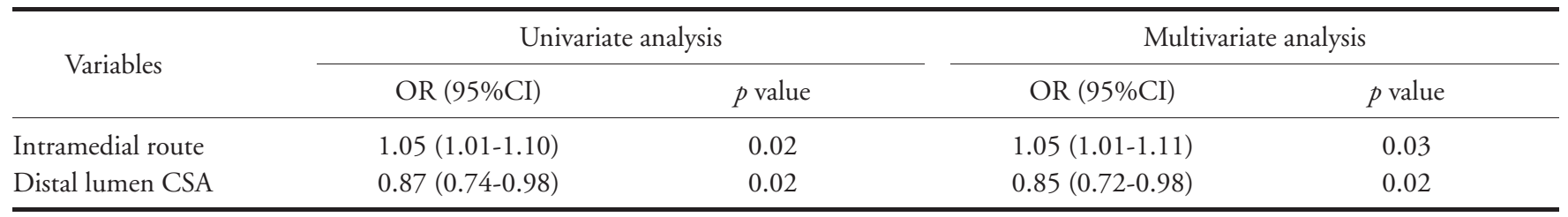

OR: odds ratio.

CI: confidence interval.

CSA: cross-sectional area.
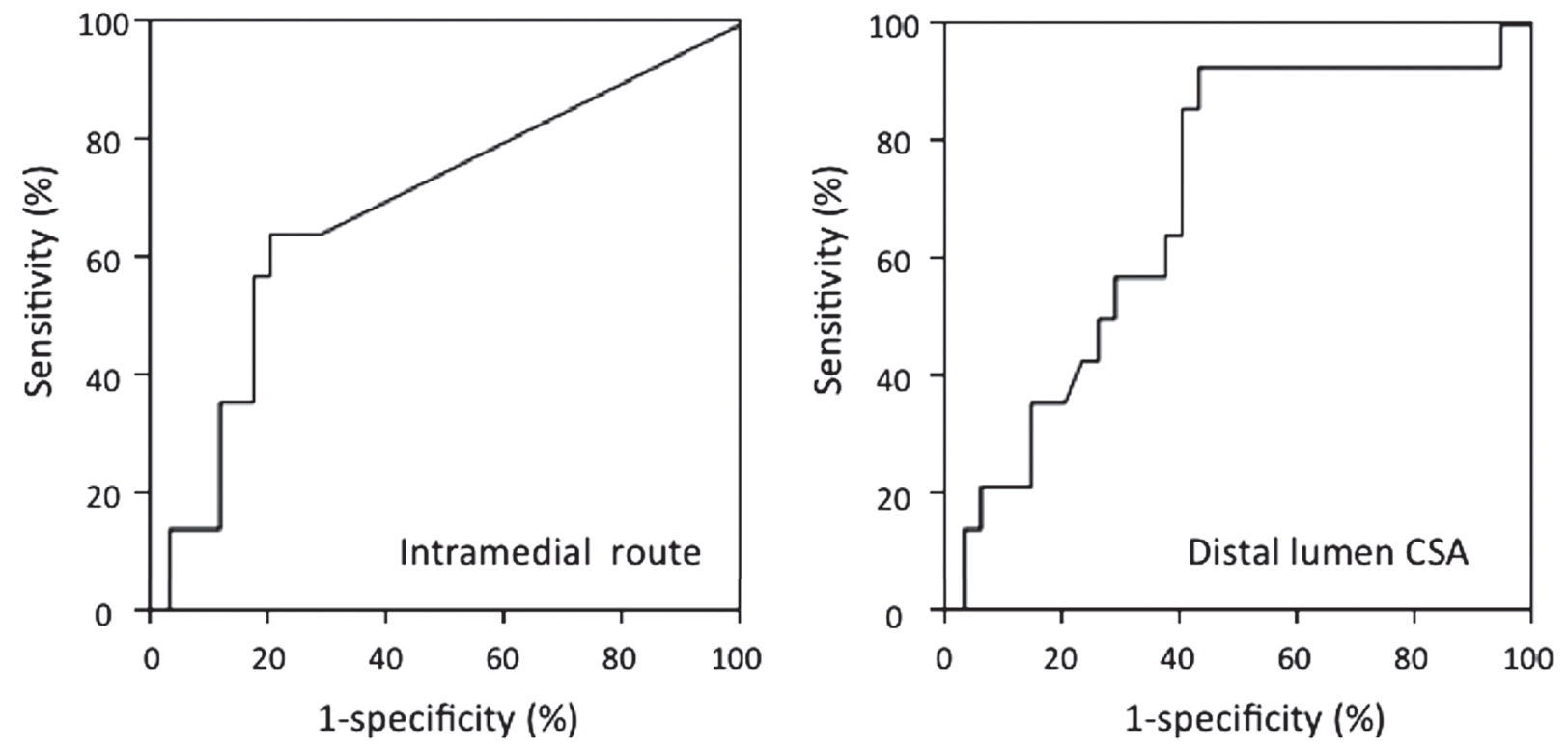

\begin{tabular}{lcccccc}
\hline & AUC & Cut-off Value & $\begin{array}{c}\text { Sensitivity } \\
\text { (\%) }\end{array}$ & $\begin{array}{c}\text { Specificity } \\
\text { (\%) }\end{array}$ & 95\% Cl & P value \\
\hline Intramedial route (\%) & 0.691 & 14.4 & 64.3 & 80.0 & $0.519-0.863$ & 0.039 \\
Distal lumen CSA (mm ${ }^{2}$ ) & 0.707 & 17.7 & 92.9 & 57.1 & $0.550-0.864$ & 0.025 \\
\hline
\end{tabular}

Fig. 2. Receiver-operating characteristic analysis of the proportion via the intramedial route and distal reference lumen CSA for developing restenosis

CSA: cross-sectional area.

AUC: area under the curve.

CI: confidence interval.

intramedial route proportion was $>14.4 \%$ in $30.6 \%$ of all lesions. On the other hand, the AUC for the distal lumen CSA was 0.707 (95\% CI $=0.550-0.864$, $p<0.05)$ and the best cutoff value of distal lumen CSA to predict restenosis was $17.7 \mathrm{~mm}^{2}$, which had a sensitivity of $92.9 \%$ and a specificity of $57.1 \%$. The distal lumen CSA was $<17.7 \mathrm{~mm}^{2}$ in $55.1 \%$ of all lesions.
Table 5. Clinical outcome at 12 months

\begin{tabular}{lc}
\hline \multicolumn{1}{c}{ Variables } & $n=49$ \\
\hline Freedom from restenosis (\%) & $35(71)$ \\
Freedom from reocclusion (\%) & $40(82)$ \\
Freedom from TLR (\%) & $40(82)$ \\
Stent thrombosis (\%) & $2(4)$ \\
\hline
\end{tabular}

TLR: target lesion revascularization 


\section{Clinical Outcomes}

Clinical outcome at 12 months are shown in Table 5. The rates of freedom from restenosis, reocclusion, and TLR were $71 \%, 82 \%$, and $82 \%$, respectively. Stent thrombosis occurred in only $2(4 \%)$ patients.

\section{Discussion}

Although there are a few reports on the clinical outcomes of the intraluminal approach for long FP lesions ${ }^{7,8,21)}$, the present study is the first to evaluate the relationship between IVUS findings and restenosis after placement of BMSs for repair of long occlusive FP lesions via the intraluminal approach.

\section{Relationship between the Within-CTO Intramedial Route Proportion and Restenosis}

Within-CTO intraplaque route proportion was significantly lower and the proportion of intramedial route was significantly higher in the restenosis group. Multivariate logistic regression analysis revealed that within-CTO intramedial route proportion was a predictor of restenosis within 12 months after EVT. The underlying reason was not investigated in the present study. However, two possible reasons can be suggested. One is that medial injury seems to contribute to restenosis. The previous study reported that a damage of internal elastic lamella was associated with increased neointimal proliferation, inflammation, and cellularity compared to de novo atherosclerosis. This correlates with a biological response of cellular proliferation and repair resulting from deep injury leading to restenosis $^{22-24)}$. The other possibility is that stent implantation via the intramedial route might cause insufficient stent expansion, leading to unfavorable short-term results, which may contribute to loss of patency. The present study also suggested that achieving a within-CTO intramedial route in $<14.4 \%$ of lesions was important and would improve primary patency. Using the subintimal approach, the guidewire was within the plaque in $<30 \%$ of lesions at the middle and distal occlusive segments ${ }^{11,25)}$. In addition, the guidewire was passed completely in the subintimal or intramedial route in $32(64 \%)$ of 50 cases, suggesting that it is difficult to maneuver the wire back to within the plaque once it enters the subintimal or intramedial space ${ }^{25)}$. The subintimal approach seems to be disadvantageous to decrease within-CTO intramedial route proportion because the maneuver of a 0.035 -inch guidewire is uncontrollable. On the other hand, by improvement in procedures and devices, the intraluminal approach enables a lower proportion of stent placements via the intramedial route; thus, there is a possibility to improve the clinical outcomes of long occlusive FP lesions. In addition, placement via the intramedial route seems to be avoided to ensure the safety of various atherectomy devices ${ }^{22)}$.

\section{Relationship between Distal Lumen CSA and Restenosis}

The distal lumen CSA was significantly smaller in the restenosis group than in the non-restenosis group and multivariate logistic regression analysis revealed that the distal lumen CSA was a predictor of restenosis within 12 months after EVT. Several studies revealed that the distal reference lumen/EEM CSA was useful to predict TLR after stent implantation for FP lesions ${ }^{16,18,19)}$, which is consistent with our results. The results of the present study suggest that restenosis risk after EVT for long occlusive lesions with small vessel is high. The ROC analysis showed that using the distal lumen CSA with a cutoff value of $17.7 \mathrm{~mm}^{2}$ could predict restenosis at 12 months after stent placement with a sensitivity of $92.9 \%$ and a specificity of $57.1 \%$ (Fig. 2). For long occlusive lesions with a distal lumen CSA of $<17.7 \mathrm{~mm}^{2}$, the primary patency at 12 months was only $55.6 \%$ in our study. This outcome is far inferior to that of autologous vein bypass grafting, which is reportedly $81 \%-88 \%$ at 12 months ${ }^{26-29)}$. However, the effectiveness of other devices, such as drug-coated balloons, atherectomy devices, and laser ablation has not been investigated for long occlusive lesions with small vessels. Consequently, at the present time, autologous vein bypass surgery should be considered more strongly for long occlusive lesions with small vessels.

\section{Limitations}

There were several limitations to this study. First, this study was retrospective and included a small number of patients. Second, each operator decided whether to use DES or BMS, and whether or not to use IVUS; thus, there was the potential for bias. Third, each within-CTO route proportion might be overestimated or underestimated because the IVUS examination was performed by manual pullback. Finally, evaluation of the IVUS findings was not performed by an independent core laboratory.

\section{Conclusion}

When the intraluminal approach was selected for treatment in long occlusive FP lesions, the whole intraplaque route was achieved in $31 \%$. If IVUS detects a proportion of the intramedial route within the CTO of $>14.4 \%$ or a distal lumen CSA of $<17.7 \mathrm{~mm}^{2}$, restenosis is more likely. 


\section{Disclosures}

This research did not receive any specific grant from funding agencies in the public, commercial, or not-for-profit sectors.

\section{References}

1) Norgren L, Hiatt WR, Dormandy JA, Nehler MR, Harris KA, Fowkes FG; TASC II Working Group: Inter-Society Consensus for the Management of Peripheral Arterial Disease (TASC II). J Vasc Surg. 2007; 45 (Suppl S): S5S67

2) Tendera M, Aboyans V, Bartelink ML, Baumqartner I, Clément D, Collet JP, Cremonesi A, De Cario M, Erbel R, Fowkes FG, Heras M, Kownator S, Minar E, Ostergren J, Poldermans D, Riambau V, Roffi M, Röther J, Sievert H, van Sambeek M, Zeller T; ESC Committee for Practice Guidelines. ESC guidelines on the diagnosis and treatment of peripheral artery disease: document covering atherosclerotic disease of extracranial carotid and vertebral, mesenteric, renal, upper and lower extremity arteries: the Task Force on the Diagnosis and Treatment of Peripheral Artery Diseases of European Society of Cardiology (ESC). Eur Heart J. 2011; 32: 2851-2906

3) Bosiers M, Peeters P, Tessarek J, Deloose K, Strickler S; Zilver PTX Single-Arm Study Investigators. The Zilver ${ }^{\circledR}$ PTX $^{\circledR}$ Zilver single arm study: 12-month results from the TASC C/D lesion subgroup. J Cardiovasc Surg. 2013; 54: $115-122$

4) Soga $Y$, Iida O, Hirano K, Yokoi $H$, Nanto $S$, Nobuyoshi M. Mid-term clinical outcome and predictors of vessel patency after femoropopliteal stenting with self-expandable nitinol stent. J Vasc Surg. 2010; 52: 608-615

5) Suzuki K, Iida O, Soga Y, Hirano K, Inoue N, Uematsu M, Nanto S, Nobuyoshi M, Meguro T. Long-term results of the S.M.A.R.T. control ${ }^{\mathrm{TM}}$ stent for superficial femoral artery lesions, J-SMART registry. Circ J. 2011; 75: 939944

6) Davaine JM, Azéma L, Guyomarch B, Chaillou P, Costargent A, Patra P, Lambert G, Gouëffic Y. One-year clinical outcome after primary stenting for Trans-Atlantic InterSociety Consensus (TASC) C and D Femoropopliteal Lesions (The STELLA "STEnting Long de L'Artere femorale superficielle" Cohort. Eur J Vasc Endovasc Surg. 2012; 44: 432-441

7) Soga Y, Iida O, Suzuki K, Hirano K, Kawasaki D, Shintani Y, Suematsu N, Yamaoka T. Initial and 3-year results after subintimal versus intraluminal approach for long femoropopliteal occlusion treated with a self-expandable nitinol stent. J Vasc Surg. 2013; 58: 1547-1555

8) Ko YG, Kim JS, Choi DH, Jang Y, Shim WH. Improve technical success and midterm patency with subintimal angioplasty compared to intraluminal angioplasty in long femoropopliteal occlusions. J Endovasc Ther. 2007; 14: 374-381

9) Baril DT, Chaer RA, Rhee RY, Makaroun MS, Marone LK. Endovascular interventions for TASC II D femoropopliteal lesions, J Vasc Surg. 2010; 51: 1406-1412

10) Tokuda T, Hirano K, Muramatsu T, Tsukahara R, Nakano
M. A sheathless retrograde approach via the popliteal artery is useful and safe for treating chronic total occlusions in the superficial femoral artery. J Endovasc Ther. 2014; 21: 289-295

11) Kawasaki D, Iida O, Fukunaga M, Kato M, Ohkubo N. Wire passage of 0.035 -inch looped wire technique for femoropopliteal long total occlusions. J Atheroscler Thromb. 2015; 22: 1071-1079

12) Cheneau E, Leborgne L, Mintz GS, Kotani J, Pichard AD, Satler LF, Canos D, Castagna M, Weissman NJ, Waksman R. Predictors of subacute stent thrombosis: result of a systematic intravascular ultrasound study. Circulation. 2003; 108: 43-47

13) Davis KL, Meyers J, Zhao Z, McCollam PL, Murakami M. High-Risk Atherosclerotic Cardiovascular Disease in a Real-World Employed Japanese Population: Prevalence, Cardiovascular Event Rates, and Costs. J Atheroscler Thromb. 2015; 22: 1287-1304

14) Takahara M, Iida O, Soda $Y$, Kodama A, Azuma N, on behalf of the SPINACH study investigators. Absence of Preceding Intermittent Claudication and its Associated Clinical Freatures in Patients with Critical Limb Ischemia. J Atheroscler Thromb. 2015; 22: 718-725

15) Soga Y, Iida O, Hirano K, Suzuki K, Tosaka A, Yokoi H, Nobuyoshi M. Utility of new classification based on clinical and lesional factors after self-expandable nitinol stenting in the superficial femoral artery. J Vasc Surg. 2011; 54: 1058-1066

16) Miki K, Fujii K, Fukunaga M, Kawasaki D, Shibuya M, Imanaka $T$, Tamaru $H$, Masutani $M$, Ohyanagi $M$, Masuyama T. Impact of post-procedural intravascular ultrasound findings on long-term results following selfexpanding nitinol stenting in superficial demoral artery lesions. Circ J. 2013; 77: 1543-1550

17) Ranke C, Creutzig A, Alexander K. Duplex scanning of the peripheral arteries: correlation of the peak velocity ratio with angiographic diameter reduction. Ultrasound Med Biol. 1992; 18: 433-440

18) Iida O, Takahara $M$, Soga $Y$, Nakano M, Yamauchi $Y$, Zen K, Kawasaki D, Nanto S, Yokoi H, Uematsu M; 1-Year results of the ZEPHYR registry (Zilver PTX for the Femoral Artery and Proximal Popliteal Artery): Predictors of restenosis. JACC Cardiovasc Interv. 2015; 8: 1105-1112

19) Mori S, Hirano K, Nakano M, Muramatsu T, Tsukahara $\mathrm{R}$, Ito $\mathrm{Y}$, Ishimori $\mathrm{H}$. Intravascular ultrasound measurement after drug-eluting stent placement in femoropopliteal lesions: determining predictors of restenosis. J Endovasc Ther. 2015; 22: 341-349

20) Mintz GS, Nissen SE, Anderson WD, Bailey SR, Erbel R, Fitzgerald PJ, Pinto FJ, Rosenfield K, Siegel RJ, Tuzcu EM, Yock PG. American College of Cardiology Clinical Expert Consensus document on standards for acquisition, measurement and reporting of Intravascular Ultrasound Studies (IVUS): A report of the American College of Cardiology Task Force on Clinical Expert Consensus Documents. J Am Coll Cardiol. 2001; 37: 1478-1492

21) Matsui J, Ochiai T, Tobita K, Shishido K, Sugitatsu K, Mizuno S, Yamanaka F, Murakami M, Tanaka Y, Takahashi S, Akasaka T, Saito S. Long-term outcome of selfexpandable nitinol stent implantation with intraluminal angioplasty to treat chronic total occlusion in the superfi- 
cial femoral artery (TransAtlantic Inter-Society Consensus Type D Lesions). J Invasive Cardiol. 2016; 28: 58-64

22) Tarricone A, Ali Z, Rajamanickam A, Gujja K, Kapur V, Purushothaman KR, Purushothaman M, Vasquez M, Zalewski A, Parides M, Overbey J, Wiley J, Krishnan P. Histopathological Evidence of Adventitial or Medial Injury Is a Strong Predictor of Restenosis During Directional Atherectomy for Peripheral Artery Disease. J Endovasc Ther. 2015; 22: 712-715

23) Pels K, Labinaz M, Hoffert C, O'Brien ER. Adventitial angiogenesis early after coronary angioplasty: correlation with arterial remodeling. Atherioscler Thromb Vasc Biol. 1999; 19: 789-796

24) Krishnan P, Purushothaman KR, Purushothaman M, Baber U, Tarricone A, Vasquez M, Wiley J, Kini A, Sharma SK, O'Connor WN, Moreno PR. Relation of Internal Elastic lamellar Layer Disruption to Neointimal Cellular Proliferation and Type III Collagen Deposition in Human Peripheral Artery Restenosis. Am J Cardiol. 2016; 117: 1173-1179

25) Takama T, Hirano K, Nakano M, Iida O, Suzuki K, Miyashita Y, Ishimori H, Ito Y, Tsukahara R, Muramatsu
T. Intravascular ultrasound findings after knuckle wire technique for superficial femoral artery occlusion. Interv Cardiol. 2014; 6: 367-371

26) Tofigh AM, Wailly DW, Rhissassi B. Comparing vein with collagen impregnated woven polyester prosthesis in above-knee femoropopliteal bypass grafting. Int J Surg. 2007; 5: 109-113

27) Klinkert P, Schepers A, Burger DH, van Bockel JH, Breslau PJ. Vein versus polytetrafluoroethylene in above knee femoro-popliteal bypass grafting: five-year results of a randomized controlled trial. J Vasc Surg. 2003; 37: 149-155

28) Johnson WC, Lee KK, and members of the Department of Veterans Affairs Study. A comparative evaluation of polytetrafluoroetylene, umbilical vein, and saphenous vein bypass graft for femoropopliteal above-knee revascularization: a prospective randomized Department of Veterans Affairs cooperative study. J Vasc Surg. 2000; 32: 268-277

29) AbuRahma AF, Robinson PA, Holt SM. Prospective controlled study of polytetrafluoroethylene versus saphenous vein in claudicant patients with bilateral above knee femoropopliteal bypass. Surgery. 1999; 126: 594-602 